\title{
A DFT Study of Pericyclic Reaction Cascades in the Synthesis of Antibiotic TAN- 1085
}

Zeve R. Akerling, Joseph E. Norton and K. N. Houk

\section{Supporting Information}

All ground state structures were fully optimized at the B3LYP/6-31G* level of theory, with Gaussian $03^{1}$. Input transition structures for parent model system A were found with potential energy surface scans at the B3LYP/STO-3G level, followed by full TS optimization at B3LYP/6-31G*. Initial transition structure approximations for model B were found through a combination of B3LYP/STO-3G potential energy surface scans and derivation from the parent model system. Structures were then optimized with B3LYP/6$31 \mathrm{G}^{*}$. All structures were validated as minima or first-degree saddle points using frequency analysis. All zero-point energy corrections are reported unscaled.

\footnotetext{
${ }^{1}$ Gaussian 03, Revision C.02, Frisch, M. J.; Trucks, G. W.; Schlegel, H. B.; Scuseria, G. E.; Robb, M. A.; Cheeseman, J. R.; Montgomery, Jr., J. A.; Vreven, T.; Kudin, K. N.; Burant, J. C.; Millam, J. M.; Iyengar, S. S.; Tomasi, J.; Barone, V.; Mennucci, B.; Cossi, M.; Scalmani, G.; Rega, N.; Petersson, G. A.; Nakatsuji, H.; Hada, M.; Ehara, M.; Toyota, K.; Fukuda, R.; Hasegawa, J.; Ishida, M.; Nakajima, T.; Honda, Y.; Kitao, O.; Nakai, H.; Klene, M.; Li, X.; Knox, J. E.; Hratchian, H. P.; Cross, J. B.; Adamo, C.; Jaramillo, J.; Gomperts, R.; Stratmann, R. E.; Yazyev, O.; Austin, A. J.; Cammi, R.; Pomelli, C.; Ochterski, J. W.; Ayala, P. Y.; Morokuma, K.; Voth, G. A.; Salvador, P.; Dannenberg, J. J.; Zakrzewski, V. G.; Dapprich, S.; Daniels, A. D.; Strain, M. C.; Farkas, O.; Malick, D. K.; Rabuck, A. D.; Raghavachari, K.; Foresman, J. B.; Ortiz, J. V.; Cui, Q.; Baboul, A. G.; Clifford, S.; Cioslowski, J.; Stefanov, B. B.; Liu, G.; Liashenko, A.; Piskorz, P.; Komaromi, I.; Martin, R. L.; Fox, D. J.; Keith, T.; Al-Laham, M. A.; Peng, C. Y.; Nanayakkara, A.; Challacombe, M.; Gill, P. M. W.; Johnson, B.; Chen, W.; Wong, M. W.; Gonzalez, C.; and Pople, J. A.; Gaussian, Inc., Pittsburgh PA, 2003
} 
Minima and transition structure geometries and energies. Energies are in Hartrees, distances and coordinates in units of Angstroms.

Model System A

$3 \mathrm{~A}$

$\begin{array}{lrrr}\mathrm{C} & -3.46124400 & 0.35486400 & -0.04799300 \\ \mathrm{C} & -2.86364400 & -0.83443300 & 0.39711600 \\ \mathrm{C} & -1.47749400 & -0.88062400 & 0.33008600 \\ \mathrm{C} & -0.72550300 & 0.19320200 & -0.13450900 \\ \mathrm{C} & -1.29789200 & 1.37855900 & -0.57340000 \\ \mathrm{C} & -2.69878600 & 1.43273500 & -0.52469800 \\ \mathrm{H} & -4.54433300 & 0.44756400 & -0.02568900 \\ \mathrm{H} & -3.46910700 & -1.65994100 & 0.76291800 \\ \mathrm{H} & -0.71542300 & 2.22133100 & -0.93521400 \\ \mathrm{H} & -3.21082100 & 2.33131700 & -0.85976500 \\ \mathrm{C} & 0.61439400 & -0.50260300 & 0.12628300 \\ \mathrm{C} & -0.26354100 & -1.75381900 & 0.60035200 \\ \mathrm{H} & -0.11077900 & -2.08268300 & 1.63499800 \\ \mathrm{H} & -0.16010800 & -2.61598700 & -0.06715500 \\ \mathrm{C} & 1.44572900 & 0.13059100 & 1.22731700 \\ \mathrm{H} & 1.24277900 & -0.25005900 & 2.22748200 \\ \mathrm{C} & 2.34156200 & 1.11951500 & 1.10986700 \\ \mathrm{H} & 2.82100500 & 1.45863000 & 2.02953300 \\ \mathrm{C} & 2.76658800 & 1.84664700 & -0.13533900 \\ \mathrm{H} & 2.56511500 & 2.92194700 & -0.03350300 \\ \mathrm{H} & 3.85005500 & 1.75059500 & -0.29205800 \\ \mathrm{H} & 2.25755000 & 1.47294500 & -1.02493900 \\ \mathrm{O} & 1.31830600 & -0.71632200 & -1.09040300 \\ \mathrm{C} & 2.41913000 & -1.60678400 & -0.99517500 \\ \mathrm{H} & 3.20092600 & -1.22740500 & -0.32474200 \\ \mathrm{H} & 2.10675400 & -2.60096200 & -0.64232000 \\ \mathrm{H} & 2.82415800 & -1.70381300 & -2.00575800\end{array}$

Zero-point correction $=0.228780$

Sum of electronic and zero-point Energies = -540.626300

TS3A

$\begin{array}{lrrr}\mathrm{C} & 3.39147000 & -0.35955200 & -0.48144400 \\ \mathrm{C} & 2.66658700 & -1.16338500 & 0.38575200 \\ \mathrm{C} & 1.33537000 & -0.79098100 & 0.67891000 \\ \mathrm{C} & 0.72647900 & 0.23607900 & -0.07287900 \\ \mathrm{C} & 1.47884200 & 1.09609800 & -0.89130800 \\ \mathrm{C} & 2.81864000 & 0.78422100 & -1.08595500 \\ \mathrm{H} & 4.43281700 & -0.59558600 & -0.68693600 \\ \mathrm{H} & 3.13069800 & -2.01480200 & 0.87771000 \\ \mathrm{H} & 1.01908200 & 1.92273200 & -1.42780200 \\ \mathrm{H} & 3.42392500 & 1.39311500 & -1.75199200 \\ \mathrm{C} & -0.72275800 & 0.08057900 & 0.08171900 \\ \mathrm{C} & 0.38604900 & -1.25674500 & 1.64275600 \\ \mathrm{H} & 0.36344500 & -2.29038200 & 1.98929100\end{array}$




$\begin{array}{lrrr}\mathrm{H} & -0.03278500 & -0.54013000 & 2.33973900 \\ \mathrm{C} & -1.40779200 & -1.04013600 & -0.55661700 \\ \mathrm{H} & -0.71513600 & -1.81393600 & -0.87344600 \\ \mathrm{C} & -2.71503800 & -1.22491000 & -0.83877100 \\ \mathrm{H} & -2.94249600 & -2.14590300 & -1.37622700 \\ \mathrm{C} & -3.91540000 & -0.37901700 & -0.52431300 \\ \mathrm{H} & -4.67762800 & -0.97978200 & -0.00810000 \\ \mathrm{H} & -3.67483800 & 0.48361500 & 0.09504600 \\ \mathrm{H} & -4.38782100 & -0.01544400 & -1.44892200 \\ \mathrm{O} & -1.51483700 & 1.14477700 & 0.35970400 \\ \mathrm{C} & -0.94028000 & 2.29430600 & 0.98646300 \\ \mathrm{H} & -0.17220300 & 2.00744000 & 1.71111100 \\ \mathrm{H} & -0.49877000 & 2.96746500 & 0.24430300 \\ \mathrm{H} & -1.76259900 & 2.80404600 & 1.49270600\end{array}$

Zero-point correction $=0.225906$

Sum of electronic and zero-point Energies $=-540.584375$

4A

$\begin{array}{lrrr}\mathrm{C} & 3.20106900 & -0.32133500 & 0.43109900 \\ \mathrm{H} & 4.24061100 & -0.32064300 & 0.74873500 \\ \mathrm{C} & 2.46098900 & -1.56154800 & 0.42275400 \\ \mathrm{H} & 2.95957200 & -2.48159900 & 0.71591900 \\ \mathrm{C} & 2.60786300 & 0.82344900 & 0.02414300 \\ \mathrm{H} & 3.16857700 & 1.75446100 & -0.01042200 \\ \mathrm{C} & 1.22624000 & 0.86781700 & -0.45696900 \\ \mathrm{C} & 1.14877500 & -1.57929400 & 0.08354700 \\ \mathrm{C} & 0.41276400 & -0.36715700 & -0.25866800 \\ \mathrm{C} & 0.82401700 & 1.98519300 & -1.11463500 \\ \mathrm{H} & -0.15326400 & 2.08364900 & -1.56942100 \\ \mathrm{H} & 1.50936200 & 2.81968000 & -1.23417900 \\ \mathrm{C} & -0.95783100 & -0.46921000 & -0.40099000 \\ \mathrm{H} & 0.59096100 & -2.50662900 & 0.10913000 \\ \mathrm{C} & -1.99634500 & 0.55792900 & -0.52441800 \\ \mathrm{H} & -2.75483600 & 0.31189600 & -1.26939600 \\ \mathrm{C} & -2.20547400 & 1.65447700 & 0.23005000 \\ \mathrm{H} & -3.08105400 & 2.25625700 & -0.01315000 \\ \mathrm{C} & -1.33307800 & 2.17176400 & 1.32500100 \\ \mathrm{H} & -0.74259600 & 1.37542500 & 1.78889800 \\ \mathrm{H} & -0.60974400 & 2.89740300 & 0.91708800 \\ \mathrm{H} & -1.92079500 & 2.68261900 & 2.09564300 \\ \mathrm{O} & -1.45689100 & -1.75508300 & -0.52618700 \\ \mathrm{C} & -2.55996200 & -2.11786200 & 0.30416100 \\ \mathrm{H} & -3.42345400 & -1.45919000 & 0.16043000 \\ \mathrm{H} & -2.27331200 & -2.10161600 & 1.36394400 \\ \mathrm{H} & -2.82906300 & -3.13639000 & 0.01582100\end{array}$

Zero-point correction $=0.227244$

Sum of electronic and zero-point Energies $=-540.611213$

$5 \mathrm{~A}$

$\begin{array}{llll}\mathrm{C} & 2.47919100 & 0.67041200 & -0.27605800\end{array}$ 


$\begin{array}{lrrr}\mathrm{C} & 1.08803000 & 0.64655900 & -0.37702300 \\ \mathrm{C} & 0.39819300 & -0.54645800 & -0.07337900 \\ \mathrm{C} & 1.11646900 & -1.68800100 & 0.30659800 \\ \mathrm{C} & 2.50768200 & -1.64940100 & 0.39541000 \\ \mathrm{C} & 3.19198100 & -0.46828100 & 0.10777100 \\ \mathrm{H} & 3.01173900 & 1.59066000 & -0.50684000 \\ \mathrm{H} & 0.57609400 & -2.59949500 & 0.53674600 \\ \mathrm{H} & 3.05475100 & -2.54008200 & 0.69310400 \\ \mathrm{H} & 4.27578900 & -0.43079700 & 0.18066900 \\ \mathrm{C} & 0.29540800 & 1.83847100 & -0.86669000 \\ \mathrm{H} & 0.86793100 & 2.76293100 & -0.72218500 \\ \mathrm{H} & 0.14489400 & 1.72885000 & -1.95194700 \\ \mathrm{C} & -1.08855400 & 1.95216800 & -0.19908700 \\ \mathrm{C} & -1.07708900 & -0.54272300 & -0.13990400 \\ \mathrm{C} & -1.78144900 & 0.60434900 & -0.20885100 \\ \mathrm{H} & -2.86637600 & 0.59736300 & -0.19735200 \\ \mathrm{H} & -1.68605200 & 2.64919000 & -0.80222200 \\ \mathrm{O} & -1.60797900 & -1.80290600 & -0.08514900 \\ \mathrm{C} & -3.01923100 & -1.92176900 & -0.07974800 \\ \mathrm{H} & -3.46046600 & -1.41960500 & 0.79181000 \\ \mathrm{H} & -3.45859000 & -1.50020100 & -0.99383700 \\ \mathrm{H} & -3.23388600 & -2.99120400 & -0.03251000 \\ \mathrm{C} & -1.00913000 & 2.52962000 & 1.22930300 \\ \mathrm{H} & -0.39053200 & 1.89445900 & 1.87292800 \\ \mathrm{H} & -0.57485000 & 3.53754900 & 1.22176000 \\ \mathrm{H} & -2.00562200 & 2.59396500 & 1.68101300\end{array}$

Zero-point correction $=0.231997$

Sum of electronic and zero-point Energies $=-540.688556$

\section{TS5A}

$\begin{array}{lrrr}\mathrm{C} & -3.29549000 & -0.01491900 & -0.24627000 \\ \mathrm{H} & -4.35198800 & -0.09729900 & -0.48799100 \\ \mathrm{C} & -2.80094800 & 1.16578400 & 0.37882700 \\ \mathrm{H} & -3.48686900 & 1.97047100 & 0.63039300 \\ \mathrm{C} & -2.44936200 & -1.06205500 & -0.49343400 \\ \mathrm{H} & -2.83563900 & -1.99511000 & -0.89770900 \\ \mathrm{C} & -1.07005600 & -1.03527200 & -0.08793100 \\ \mathrm{C} & -1.46115800 & 1.30581700 & 0.62241100 \\ \mathrm{C} & -0.52618900 & 0.26654400 & 0.28216600 \\ \mathrm{C} & -0.35488200 & -2.22973700 & -0.05606000 \\ \mathrm{H} & -0.77398700 & -3.09564600 & -0.56720100 \\ \mathrm{H} & 0.28036600 & -2.46423800 & 0.77581500 \\ \mathrm{C} & 0.79811000 & 0.65100600 & -0.04736400 \\ \mathrm{H} & -1.07227700 & 2.23641900 & 1.01838100 \\ \mathrm{C} & 1.73245000 & -0.16859000 & -0.72864600 \\ \mathrm{H} & 2.13738900 & 0.26488800 & -1.64608200 \\ \mathrm{C} & 2.03717600 & -1.48417800 & -0.44535100 \\ \mathrm{H} & 2.52552000 & -2.04088400 & -1.24668900 \\ \mathrm{C} & 2.39613900 & -1.97895000 & 0.93711800 \\ \mathrm{H} & 2.21398300 & -3.05133700 & 1.06846600 \\ \mathrm{H} & 3.47415000 & -1.81974000 & 1.09250300 \\ \mathrm{H} & 1.87081800 & -1.42822100 & 1.72316600 \\ \mathrm{O} & 1.00893300 & 2.00793100 & -0.00297000 \\ \mathrm{C} & 2.32288000 & 2.50256300 & -0.22002900\end{array}$




$\begin{array}{lrrr}\mathrm{H} & 3.07378900 & 1.87032400 & 0.26914000 \\ \mathrm{H} & 2.34346400 & 3.50771000 & 0.20870800 \\ \mathrm{H} & 2.55779400 & 2.57113900 & -1.28976000\end{array}$

Zero-point correction=

Sum of electronic and zero-point Energies=

0.227138

$-540.589398$

$6 \mathrm{~A}$

$\begin{array}{lrrr}\mathrm{C} & -0.95890500 & -1.13819200 & -1.04838500 \\ \mathrm{C} & -2.34643700 & -1.22649700 & -1.13881900 \\ \mathrm{C} & -3.13623000 & -0.54155800 & -0.21587600 \\ \mathrm{C} & -2.53082800 & 0.21546200 & 0.78685100 \\ \mathrm{C} & -1.13797400 & 0.32172800 & 0.89170000 \\ \mathrm{C} & -0.34535700 & -0.35801300 & -0.05771200 \\ \mathrm{H} & -0.33259700 & -1.67484300 & -1.75553200 \\ \mathrm{H} & -2.80455700 & -1.82801800 & -1.91922300 \\ \mathrm{H} & -4.22020400 & -0.60213900 & -0.26888300 \\ \mathrm{H} & -3.15095500 & 0.73341400 & 1.51497500 \\ \mathrm{C} & 1.14556400 & -0.34101800 & -0.00367100 \\ \mathrm{C} & -0.52995700 & 1.15226600 & 1.99687300 \\ \mathrm{H} & -1.28345600 & 1.40459800 & 2.74968000 \\ \mathrm{H} & 0.28925300 & 0.62522500 & 2.49723000 \\ \mathrm{H} & -0.11743900 & 2.08841900 & 1.60246900 \\ \mathrm{O} & 1.59529200 & -1.59380500 & 0.32443800 \\ \mathrm{C} & 2.99397800 & -1.83635400 & 0.34419900 \\ \mathrm{H} & 3.45306800 & -1.60347600 & -0.62541800 \\ \mathrm{H} & 3.49290800 & -1.24913300 & 1.12645000 \\ \mathrm{H} & 3.10943000 & -2.89991600 & 0.56161000 \\ \mathrm{C} & 1.95967800 & 0.71281800 & -0.24463800 \\ \mathrm{H} & 3.02598600 & 0.56712500 & -0.09562700 \\ \mathrm{C} & 1.59867300 & 2.06335200 & -0.68177700 \\ \mathrm{H} & 2.36165000 & 2.81026600 & -0.45660700 \\ \mathrm{C} & 0.50680800 & 2.48228400 & -1.34180000 \\ \mathrm{H} & 0.38997100 & 3.53027700 & -1.60284000 \\ \mathrm{H} & -0.28946900 & 1.81097300 & -1.64546700\end{array}$

Zero-point correction $=0.228312$

Sum of electronic and zero-point Energies $=-540.652704$

TS6A

$\begin{array}{rrrr}\mathrm{C} & 3.17981600 & -0.59168500 & 0.26097900 \\ \mathrm{H} & 4.25122400 & -0.66380700 & 0.42972600 \\ \mathrm{C} & 2.34269100 & -1.71149300 & 0.51140700 \\ \mathrm{H} & 2.77109100 & -2.63776500 & 0.88456400 \\ \mathrm{C} & 2.63298900 & 0.56979200 & -0.21086900 \\ \mathrm{H} & 3.27112600 & 1.42120700 & -0.43556000 \\ \mathrm{C} & 1.23014600 & 0.70821100 & -0.48608200 \\ \mathrm{C} & 0.99099900 & -1.61372900 & 0.31221400 \\ \mathrm{C} & 0.36144300 & -0.39709600 & -0.12186600 \\ \mathrm{C} & 0.78972600 & 1.91071200 & -1.07251100 \\ \mathrm{H} & -0.13421600 & 1.91877700 & -1.64708400 \\ \mathrm{H} & 1.54752800 & 2.61622300 & -1.40694900 \\ \mathrm{C} & -1.07095300 & -0.40789100 & -0.20955900\end{array}$




$\begin{array}{rrrr}\mathrm{H} & 0.35357900 & -2.46090100 & 0.53144200 \\ \mathrm{C} & -2.02459600 & 0.62881900 & -0.10911000 \\ \mathrm{H} & -3.01249600 & 0.35812000 & -0.47351900 \\ \mathrm{C} & -1.94625900 & 1.90271100 & 0.43997700 \\ \mathrm{H} & -2.82810100 & 2.52589600 & 0.29827000 \\ \mathrm{C} & -0.79527400 & 2.51203800 & 1.02160000 \\ \mathrm{H} & -0.17865300 & 1.86228000 & 1.65229500 \\ \mathrm{H} & 0.03405600 & 2.59368100 & 0.08394200 \\ \mathrm{H} & -0.95314000 & 3.49526700 & 1.46691900 \\ \mathrm{O} & -1.55071500 & -1.68298100 & -0.42538100 \\ \mathrm{C} & -2.87165100 & -2.03170700 & -0.03244500 \\ \mathrm{H} & -3.61556800 & -1.71070800 & -0.77281300 \\ \mathrm{H} & -3.12783200 & -1.60344400 & 0.94384300 \\ \mathrm{H} & -2.88735100 & -3.12306600 & 0.02556000\end{array}$

Zero-point correction $=0.224719$

Sum of electronic and zero-point Energies $=-540.603248$

Model System B

$3 B$

\begin{tabular}{|c|c|c|c|}
\hline $\mathrm{C}$ & 3.11452400 & -1.84609600 & 0.22492300 \\
\hline $\mathrm{C}$ & 2.78130300 & -0.49750000 & 0.40850900 \\
\hline $\mathrm{C}$ & 1.44931000 & -0.16502900 & 0.18716700 \\
\hline $\mathrm{C}$ & 0.49430700 & -1.11141300 & -0.15868000 \\
\hline $\mathrm{C}$ & 0.80676200 & -2.45323500 & -0.33638400 \\
\hline $\mathrm{C}$ & 2.15106700 & -2.79898100 & -0.14608000 \\
\hline $\mathrm{H}$ & 4.14214200 & -2.16790400 & 0.37382600 \\
\hline $\mathrm{H}$ & 3.53153400 & 0.22828800 & 0.70918900 \\
\hline $\mathrm{H}$ & 0.06620800 & -3.20187900 & -0.60263100 \\
\hline $\mathrm{H}$ & 2.45995500 & -3.83314000 & -0.27704200 \\
\hline $\mathrm{C}$ & -0.68970300 & -0.14601600 & -0.12322100 \\
\hline $\mathrm{C}$ & 0.45993400 & 0.99020500 & 0.16495400 \\
\hline $\mathrm{C}$ & -1.60376300 & -0.39419100 & 1.06084400 \\
\hline $\mathrm{H}$ & -1.25967700 & 0.06182100 & 1.98584900 \\
\hline $\mathrm{C}$ & -2.70404900 & -1.15766300 & 1.10815400 \\
\hline $\mathrm{H}$ & -3.20174200 & -1.23233800 & 2.07653100 \\
\hline $\mathrm{C}$ & -3.34278800 & -1.95551100 & 0.00552000 \\
\hline $\mathrm{H}$ & -3.40828500 & -3.01343100 & 0.29488000 \\
\hline $\mathrm{H}$ & -4.37492500 & -1.62208300 & -0.17254300 \\
\hline $\mathrm{H}$ & -2.78956200 & -1.87971800 & -0.93151500 \\
\hline O & -1.32800800 & -0.07717600 & -1.37936000 \\
\hline $\mathrm{C}$ & -2.29242700 & 0.95794000 & -1.53605500 \\
\hline $\mathrm{H}$ & -3.11996700 & 0.85844800 & -0.82225700 \\
\hline $\mathrm{H}$ & -1.83227700 & 1.94590900 & -1.42955500 \\
\hline $\mathrm{H}$ & -2.68192700 & 0.85254800 & -2.55207300 \\
\hline $\mathrm{O}$ & 0.43251800 & 1.68339500 & 1.38685600 \\
\hline $\mathrm{O}$ & 0.54201900 & 1.85790100 & -0.93304200 \\
\hline $\mathrm{C}$ & -0.52947300 & 2.73353300 & 1.47121600 \\
\hline $\mathrm{H}$ & -0.46706800 & 3.11709900 & 2.49235700 \\
\hline $\mathrm{H}$ & -0.31285400 & 3.54062100 & 0.76323300 \\
\hline $\mathrm{H}$ & -1.54660400 & 2.36440600 & 1.28636900 \\
\hline $\mathrm{C}$ & 1.78908200 & 2.53181500 & -1.06877800 \\
\hline $\mathrm{H}$ & 2.59491400 & 1.82456000 & -1.30397300 \\
\hline
\end{tabular}




$\begin{array}{llll}\mathrm{H} & 1.67190200 & 3.23088200 & -1.90003900 \\ \mathrm{H} & 2.05148600 & 3.08579600 & -0.15877500\end{array}$

Zero-point correction $=0.294275$

Sum of electronic and zero-point Energies $=-769.602573$

\section{TS3B}

\begin{tabular}{|c|c|c|c|}
\hline $\mathrm{C}$ & -3.05292900 & -1.39400700 & -0.89198600 \\
\hline $\mathrm{C}$ & -2.57365800 & -0.11018200 & -0.68165100 \\
\hline $\mathrm{C}$ & -1.24421900 & 0.03434100 & -0.22297100 \\
\hline C & -0.37638000 & -1.07923000 & -0.19757100 \\
\hline $\mathrm{C}$ & -0.89670800 & -2.38250000 & -0.32381500 \\
\hline $\mathrm{C}$ & -2.23079100 & -2.52563200 & -0.67812300 \\
\hline $\mathrm{H}$ & -4.08956600 & -1.53985600 & -1.18489200 \\
\hline $\mathrm{H}$ & -3.23281100 & 0.74836600 & -0.77757100 \\
\hline $\mathrm{H}$ & -0.25032900 & -3.25296500 & -0.24643800 \\
\hline $\mathrm{H}$ & -2.64162200 & -3.51846200 & -0.84137700 \\
\hline $\mathrm{C}$ & 1.01046500 & -0.64334200 & -0.13993200 \\
\hline $\mathrm{C}$ & -0.54868800 & 1.18206200 & 0.31493800 \\
\hline $\mathrm{C}$ & 1.61851600 & 0.04738400 & -1.26323000 \\
\hline $\mathrm{H}$ & 0.90088000 & 0.27442700 & -2.04792200 \\
\hline $\mathrm{C}$ & 2.90928000 & 0.38300300 & -1.49570100 \\
\hline $\mathrm{H}$ & 3.10359200 & 0.84291700 & -2.46494200 \\
\hline $\mathrm{C}$ & 4.11684600 & 0.23855800 & -0.61470100 \\
\hline $\mathrm{H}$ & 3.86044400 & -0.08523700 & 0.39335700 \\
\hline $\mathrm{H}$ & 4.82844700 & -0.49000100 & -1.03244100 \\
\hline $\mathrm{H}$ & 4.65808800 & 1.19319800 & -0.54973700 \\
\hline 0 & 1.89147700 & -1.24110200 & 0.70740700 \\
\hline $\mathrm{C}$ & 1.40145900 & -1.77275500 & 1.94118700 \\
\hline $\mathrm{H}$ & 0.64635000 & -1.11011100 & 2.37049500 \\
\hline $\mathrm{H}$ & 0.98184000 & -2.77578600 & 1.80515600 \\
\hline $\mathrm{H}$ & 2.26771800 & -1.83568600 & 2.60385000 \\
\hline $\mathrm{O}$ & -0.12654300 & 1.18154000 & 1.62403000 \\
\hline O & -0.84240900 & 2.45879600 & -0.07554000 \\
\hline $\mathrm{C}$ & -1.09536400 & 1.69993700 & 2.55691000 \\
\hline $\mathrm{H}$ & -2.03375800 & 1.13273500 & 2.49816800 \\
\hline $\mathrm{H}$ & -0.65189200 & 1.57590900 & 3.54713800 \\
\hline $\mathrm{H}$ & -1.29237400 & 2.75962500 & 2.37040600 \\
\hline $\mathrm{C}$ & -0.95379500 & 2.72757800 & -1.47227300 \\
\hline $\mathrm{H}$ & -1.09319800 & 3.80726900 & -1.55750300 \\
\hline $\mathrm{H}$ & -0.03716200 & 2.43228000 & -1.99236600 \\
\hline $\mathrm{H}$ & -1.80904900 & 2.21622600 & -1.92705000 \\
\hline
\end{tabular}

Zero-point correction $=0.291543$

Sum of electronic and zero-point Energies $=-769.560077$

4B

$\begin{array}{rrrr}\mathrm{C} & -0.18011700 & 3.22369800 & 0.49495500 \\ \mathrm{H} & -0.50964200 & 4.18623700 & 0.87744800 \\ \mathrm{C} & 1.10903400 & 3.11830000 & -0.14920600 \\ \mathrm{H} & 1.70834100 & 4.01433800 & -0.28946900 \\ \mathrm{C} & -0.98876800 & 2.14059100 & 0.58696900 \\ \mathrm{H} & -1.98790300 & 2.22618800 & 0.99899600\end{array}$




$\begin{array}{lrrr}\text { C } & -0.58642900 & 0.85312600 & 0.02640800 \\ \mathrm{C} & 1.59276200 & 1.91067200 & -0.53446300 \\ \mathrm{C} & 0.85498900 & 0.67747300 & -0.28254300 \\ \mathrm{C} & -1.56862700 & -0.02476000 & -0.33350000 \\ \mathrm{C} & 1.52914900 & -0.52185400 & -0.31745000 \\ \mathrm{H} & 2.58400200 & 1.82192300 & -0.96274600 \\ \mathrm{C} & 1.14000100 & -1.83454200 & 0.20414100 \\ \mathrm{H} & 1.35687100 & -2.66127200 & -0.47456700 \\ \mathrm{C} & 0.65829700 & -2.14236100 & 1.42135300 \\ \mathrm{H} & 0.46296300 & -3.19624300 & 1.62075300 \\ \mathrm{C} & 0.33681900 & -1.19864500 & 2.53910700 \\ \mathrm{H} & 0.79223300 & -0.21628000 & 2.38827500 \\ \mathrm{H} & -0.74950300 & -1.04386400 & 2.62185900 \\ \mathrm{H} & 0.67325900 & -1.60497300 & 3.50136500 \\ \mathrm{O} & 2.75494100 & -0.51793600 & -0.96968300 \\ \mathrm{C} & 3.86636700 & -1.05343300 & -0.25494400 \\ \mathrm{H} & 3.70147100 & -2.09382900 & 0.04843600 \\ \mathrm{H} & 4.08330100 & -0.45509800 & 0.64034600 \\ \mathrm{H} & 4.71754900 & -1.00559800 & -0.93845000 \\ \mathrm{O} & -1.32847300 & -1.22180300 & -0.92637300 \\ \mathrm{O} & -2.88929600 & 0.29220200 & -0.15845700 \\ \mathrm{C} & -1.99661900 & -1.42711400 & -2.18221100 \\ \mathrm{H} & -3.08518300 & -1.42615400 & -2.06867500 \\ \mathrm{H} & -1.70722200 & -0.65050200 & -2.89923800 \\ \mathrm{H} & -1.66283300 & -2.40335800 & -2.53805600 \\ \mathrm{C} & -3.63036500 & -0.61979800 & 0.66325500 \\ \mathrm{H} & -3.55723600 & -1.64779500 & 0.29317600 \\ \mathrm{H} & -3.26718600 & -0.58453800 & 1.69840300 \\ \mathrm{H} & -4.66961300 & -0.28701900 & 0.62702300\end{array}$

Zero-point correction $=0.292455$

Sum of electronic and zero-point Energies $=-769.589449$

$5 B$

$\begin{array}{lrrr}\text { C } & 0.82118900 & 2.12193700 & -0.39780900 \\ \mathrm{C} & 0.29962500 & 0.84940300 & -0.16703700 \\ \mathrm{C} & -1.09838200 & 0.67408300 & -0.08435400 \\ \mathrm{C} & -1.94038300 & 1.78774600 & -0.20416200 \\ \mathrm{C} & -1.40648200 & 3.05745100 & -0.42073900 \\ \mathrm{C} & -0.02609000 & 3.22522800 & -0.52801700 \\ \mathrm{H} & 1.89581000 & 2.24076500 & -0.47738800 \\ \mathrm{H} & -3.01305700 & 1.64551400 & -0.13661200 \\ \mathrm{H} & -2.07076400 & 3.91262500 & -0.51467300 \\ \mathrm{H} & 0.39400300 & 4.21105000 & -0.70928000 \\ \mathrm{C} & 1.19239600 & -0.36421200 & 0.08246300 \\ \mathrm{C} & 0.57494500 & -1.63657900 & -0.54280300 \\ \mathrm{C} & -1.64350700 & -0.68804800 & 0.07058200 \\ \mathrm{C} & -0.87574000 & -1.77307100 & -0.13435700 \\ \mathrm{H} & -1.28336100 & -2.77610900 & -0.07296500 \\ \mathrm{H} & 1.12824900 & -2.48342100 & -0.11901900 \\ \mathrm{O} & -2.97402100 & -0.69349500 & 0.39012900 \\ \mathrm{C} & -3.61338500 & -1.95289700 & 0.49751400 \\ \mathrm{H} & -3.56919000 & -2.50546200 & -0.45075400 \\ \mathrm{H} & -3.15851000 & -2.56432600 & 1.28824400 \\ \mathrm{H} & -4.65467100 & -1.74472500 & 0.75110500\end{array}$




$\begin{array}{rrrr}\mathrm{C} & 0.71243300 & -1.66828200 & -2.07878200 \\ \mathrm{H} & 0.18300400 & -0.82365700 & -2.53129500 \\ \mathrm{H} & 1.75806600 & -1.62205900 & -2.39788200 \\ \mathrm{H} & 0.27202900 & -2.59031900 & -2.47379300 \\ \mathrm{O} & 2.47173800 & -0.04924800 & -0.44476100 \\ \mathrm{O} & 1.28364400 & -0.66616400 & 1.47312800 \\ \mathrm{C} & 3.50149900 & -1.00364600 & -0.21485300 \\ \mathrm{H} & 3.55863100 & -1.29046800 & 0.84019300 \\ \mathrm{H} & 3.36687200 & -1.90906000 & -0.82141800 \\ \mathrm{H} & 4.43388700 & -0.51788200 & -0.51372600 \\ \mathrm{C} & 1.68842100 & 0.39226500 & 2.33299200 \\ \mathrm{H} & 0.91396500 & 1.16320600 & 2.42566700 \\ \mathrm{H} & 1.85665900 & -0.06600300 & 3.31111700 \\ \mathrm{H} & 2.61824400 & 0.86331500 & 1.99067600\end{array}$

Zero-point correction $=0.297154$

Sum of electronic and zero-point Energies $=-769.662263$

\section{TS5B}

\begin{tabular}{|c|c|c|c|}
\hline C & 0.62869600 & 2.36418200 & 0.06604800 \\
\hline $\mathrm{C}$ & 0.34692400 & 0.97647100 & -0.10384300 \\
\hline $\mathrm{C}$ & -1.03881900 & 0.57666300 & -0.28450600 \\
\hline C & -1.95527800 & 1.62365500 & -0.65304000 \\
\hline $\mathrm{C}$ & -1.60702400 & 2.94917800 & -0.60891900 \\
\hline $\mathrm{C}$ & -0.30917000 & 3.33721300 & -0.19475700 \\
\hline $\mathrm{H}$ & 1.65570800 & 2.65421000 & 0.27012600 \\
\hline $\mathrm{H}$ & -2.97133400 & 1.33774500 & -0.89409000 \\
\hline $\mathrm{H}$ & -2.33839700 & 3.70744300 & -0.87810200 \\
\hline $\mathrm{H}$ & -0.03053900 & 4.38665300 & -0.16017500 \\
\hline $\mathrm{C}$ & 1.50313300 & 0.12841800 & -0.09444600 \\
\hline $\mathrm{C}$ & 0.26036400 & -2.25409500 & 0.54631200 \\
\hline $\mathrm{C}$ & -1.59782900 & -0.62303000 & 0.22110100 \\
\hline $\mathrm{C}$ & -0.89582500 & -1.61927300 & 0.93840100 \\
\hline $\mathrm{H}$ & -1.31941800 & -1.85407800 & 1.92157500 \\
\hline $\mathrm{H}$ & 0.76602200 & -2.84909100 & 1.30945900 \\
\hline O & -2.97693500 & -0.57624400 & 0.42504600 \\
\hline $\mathrm{C}$ & -3.68889500 & -1.74877600 & 0.05019500 \\
\hline $\mathrm{H}$ & -3.54855900 & -1.97769600 & -1.01568200 \\
\hline $\mathrm{H}$ & -3.38630700 & -2.62397700 & 0.63904700 \\
\hline $\mathrm{H}$ & -4.74547800 & -1.53983900 & 0.24018100 \\
\hline $\mathrm{C}$ & 0.48313900 & -2.73908300 & -0.86511300 \\
\hline $\mathrm{H}$ & 0.04301500 & -2.07474700 & -1.60980200 \\
\hline $\mathrm{H}$ & 1.53395600 & -2.89841900 & -1.11258200 \\
\hline $\mathrm{H}$ & -0.00594300 & -3.72168200 & -0.96101700 \\
\hline O & 1.91692600 & -0.41908600 & -1.25238900 \\
\hline 0 & 2.53856400 & 0.38653300 & 0.74754800 \\
\hline $\mathrm{C}$ & 3.20582500 & -1.04511400 & -1.27666100 \\
\hline $\mathrm{H}$ & 3.99593100 & -0.29185200 & -1.21323200 \\
\hline $\mathrm{H}$ & 3.32417600 & -1.75845400 & -0.45524700 \\
\hline $\mathrm{H}$ & 3.25674400 & -1.56036000 & -2.23695700 \\
\hline C & 2.23408800 & 0.47987800 & 2.14508200 \\
\hline $\mathrm{H}$ & 1.70421200 & -0.42320300 & 2.46524200 \\
\hline $\mathrm{H}$ & 3.19817300 & 0.56033900 & 2.650760 \\
\hline $\mathrm{H}$ & 1.62363000 & 1.35966600 & 2.363 \\
\hline
\end{tabular}


Zero-point correction $=0.292341$

Sum of electronic and zero-point Energies $=-769.555004$

$6 B$

\begin{tabular}{|c|c|c|c|}
\hline C & 1.31620700 & 1.98425300 & -0.66874300 \\
\hline C & 0.54828100 & 3.12711900 & -0.89030700 \\
\hline $\mathrm{C}$ & -0.83343600 & 3.07108300 & -0.71397300 \\
\hline $\mathrm{C}$ & -1.43970400 & 1.87589800 & -0.32439900 \\
\hline $\mathrm{C}$ & -0.67993900 & 0.72333000 & -0.10355000 \\
\hline $\mathrm{C}$ & 0.71890900 & 0.78365800 & -0.26547400 \\
\hline $\mathrm{H}$ & 2.39419800 & 2.01658400 & -0.79982800 \\
\hline $\mathrm{H}$ & 1.02793200 & 4.05296400 & -1.19639500 \\
\hline $\mathrm{H}$ & -1.44280800 & 3.95559900 & -0.88019100 \\
\hline $\mathrm{H}$ & -2.51310300 & 1.82544400 & -0.18523600 \\
\hline $\mathrm{C}$ & 1.58846100 & -0.41535100 & -0.07500800 \\
\hline C & -1.33987800 & -0.58404700 & 0.32280000 \\
\hline $\mathrm{H}$ & -0.91390700 & -0.92245400 & 1.27648700 \\
\hline O & 1.91864500 & -0.92692700 & -1.29925200 \\
\hline C & 2.68722000 & -2.11985500 & -1.35314500 \\
\hline $\mathrm{H}$ & 3.67375100 & -1.98288700 & -0.89113600 \\
\hline $\mathrm{H}$ & 2.16437300 & -2.94609900 & -0.85552800 \\
\hline $\mathrm{H}$ & 2.81009800 & -2.34779400 & -2.41360700 \\
\hline C & 2.01086200 & -0.92068300 & 1.10619300 \\
\hline $\mathrm{H}$ & 2.61049000 & -1.82534600 & 1.07508700 \\
\hline C & 1.74013300 & -0.43658100 & 2.46219200 \\
\hline $\mathrm{H}$ & 1.98461700 & -1.17220600 & 3.22977000 \\
\hline C & 1.27144600 & 0.74802800 & 2.89009200 \\
\hline $\mathrm{H}$ & 1.13367700 & 0.92686700 & 3.95271000 \\
\hline $\mathrm{H}$ & 1.02337300 & 1.56658300 & 2.22389100 \\
\hline O & -1.07172600 & -1.66380900 & -0.55589800 \\
\hline O & -2.72170500 & -0.35838500 & 0.46986200 \\
\hline C & -1.44531400 & -1.44913800 & -1.91503500 \\
\hline $\mathrm{H}$ & -1.34976200 & -2.41830700 & -2.41119800 \\
\hline $\mathrm{H}$ & -2.48215100 & -1.10184700 & -1.99652000 \\
\hline $\mathrm{H}$ & -0.77972800 & -0.72839900 & -2.40442900 \\
\hline C & -3.40337600 & -1.42517800 & 1.11288200 \\
\hline $\mathrm{H}$ & -3.31648300 & -2.35933800 & 0.54600500 \\
\hline $\mathrm{H}$ & -3.01157600 & -1.58982300 & 2.12790700 \\
\hline $\mathrm{H}$ & -4.45394700 & -1.13180800 & 1.1773750 \\
\hline
\end{tabular}

Zero-point correction $=0.294718$

Sum of electronic and zero-point Energies $=-769.624436$

\section{TS6B}

$\begin{array}{lrrr}\mathrm{C} & -0.58153200 & 3.21571500 & -0.30976600 \\ \mathrm{H} & -0.40310300 & 4.28694000 & -0.35225600 \\ \mathrm{C} & -1.89706900 & 2.70725200 & -0.39809600 \\ \mathrm{H} & -2.73754900 & 3.38262100 & -0.53485000 \\ \mathrm{C} & 0.47251700 & 2.34942000 & -0.15538400 \\ \mathrm{H} & 1.48214500 & 2.73004100 & -0.06497000 \\ \mathrm{C} & 0.28688800 & 0.93689900 & -0.05211800 \\ \mathrm{C} & -2.10862200 & 1.35121400 & -0.35411400 \\ \mathrm{C} & -1.04249300 & 0.39929600 & -0.23756100\end{array}$




$\begin{array}{lrrr}\text { C } & 1.45845300 & 0.13178100 & 0.17373100 \\ \mathrm{C} & -1.44865600 & -0.98862300 & -0.32216200 \\ \mathrm{H} & -3.11090000 & 0.95902800 & -0.48193400 \\ \mathrm{C} & -0.82597400 & -2.11949700 & -0.84996700 \\ \mathrm{H} & -1.36884200 & -3.03844200 & -0.63606800 \\ \mathrm{C} & 0.33380600 & -2.25205100 & -1.61309400 \\ \mathrm{H} & 0.71007800 & -3.26540300 & -1.73891900 \\ \mathrm{C} & 1.10161300 & -1.18378800 & -2.15740500 \\ \mathrm{H} & 1.57333900 & -0.53348300 & -1.19319900 \\ \mathrm{O} & -2.76327500 & -1.25396700 & 0.07152600 \\ \mathrm{C} & -3.04169300 & -1.09353400 & 1.45994800 \\ \mathrm{H} & -2.86145700 & -0.06713900 & 1.80262100 \\ \mathrm{H} & -2.43643900 & -1.78562500 & 2.06161500 \\ \mathrm{H} & -4.09977500 & -1.33515100 & 1.59046600 \\ \mathrm{H} & 1.92839000 & -1.47633200 & -2.80680300 \\ \mathrm{H} & 0.52772300 & -0.34691600 & -2.56990600 \\ \mathrm{O} & 1.37324600 & -1.08148700 & 0.81486300 \\ \mathrm{O} & 2.61400500 & 0.83758700 & 0.46702400 \\ \mathrm{C} & 1.05911300 & -0.99747900 & 2.21077100 \\ \mathrm{H} & 1.81432900 & -0.40615300 & 2.74167800 \\ \mathrm{H} & 0.07144100 & -0.54878000 & 2.36433300 \\ \mathrm{H} & 1.06095800 & -2.02292700 & 2.58640500 \\ \mathrm{C} & 3.83288200 & 0.13398500 & 0.24464500 \\ \mathrm{H} & 3.85479100 & -0.81514900 & 0.78931800 \\ \mathrm{H} & 3.97705100 & -0.06476700 & -0.82602400 \\ \mathrm{H} & 4.63061400 & 0.78703900 & 0.60462000\end{array}$

Zero-point correction $=0.289707$

Sum of electronic and zero-point Energies $=-769.573315$ 